\title{
Aspirin and heparin in the treatment of recurrent spontaneous abortion associated with antiphospholipid antibody syndrome: A systematic review and meta-analysis
}

\author{
XIAOMEI YU ${ }^{1}$ and $\mathrm{LI} \mathrm{HE}^{2}$ \\ ${ }^{1}$ Department of Obstetrics, Ward 1, Weifang People's Hospital, Weifang, Shandong 261041; \\ ${ }^{2}$ Department of Women's Health Care, Chongqing Health Center for Women and Children, \\ Yubei, Chongqing 401147, P.R. China
}

Received June 24, 2019; Accepted March 17, 2020

DOI: $10.3892 /$ etm.2020.9489

\begin{abstract}
The present study aimed to review relevant, randomized, controlled trials in order to determine the effects of aspirin and heparin treatment on recurrent spontaneous abortion (RSA) in women with antiphospholipid syndrome (APS). Previous relevant studies were identified using PubMed, Cochrane, Embase, CNKI, VANFUN and VIP by retrieving appropriate key words. Additionally, key relevant sources in the literature were reviewed and articles published before May 2019 were included. The 22 selected studies included 1,515 patients in the treatment group and 1,531 patients in the control group. These previous studies showed that heparin and aspirin significantly improved live birth rate when compared with treatments using intravenous immunoglobulin, aspirin alone or aspirin combined with prednisone. Moreover, heparin and aspirin greatly increased the birth weight compared with placebo and improved vaginal delivery relative to intravenous immunoglobulin. The gestational age at birth was significantly higher in the heparin and aspirin group compared with the placebo group and the incidence of intrauterine growth restriction was lower in the heparin and aspirin group compared with the placebo group. Furthermore, heparin and aspirin markedly reduced the incidence of miscarriage compared with the aspirin group and the placebo group, and the incidence of pre-eclampsia was lower in the heparin and aspirin group than the placebo group. Thus, heparin and aspirin could be further examined for the treatment of RSA in women with APS.
\end{abstract}

\section{Introduction}

Antiphospholipid syndrome (APS) is an autoimmune disease associated with arterial or venous thrombosis, recurrent fetal

Correspondence to: Dr Li He, Department of Women's Health Care, Chongqing Health Center for Women and Children, 120 Longshan Road, Yubei, Chongqing 401147, P.R. China

E-mail: helih_11@163.com

Key words: aspirin, heparin, recurrent spontaneous abortion, antiphospholipid syndrome, meta-analysis abortion or thrombocytopenia (1). The development of APS involves the production of antiphospholipid antibodies, such as anticardiolipin antibodies (ACL), lupus anticoagulant (LA) and anti- $\beta 2$ glycoprotein I antibodies $(2,3)$. The major pathological changes seen in APS caused by antibodies include villus microtubule embolism, placental infarction and fetal arterial embolism, which can result in embryo abortion and stillbirth (4,5). Termination of pregnancy at $<28$ weeks or fetal weight $<1 \mathrm{~kg}$ is referred to as abortion and recurrent spontaneous abortion (RSA) is defined as continuous spontaneous abortion occurring more than twice (6). RSA is a common phenomenon with an incidence rate of $1-3 \%$ (7). Risk factors for RSA range from genetic, hormonal or metabolic factors, to uterine anatomy, autoimmune dysfunction, thrombosis tendency and infection (8). APS accounts for 7-25\% of RSA and the rate of miscarriage can reach $90 \%$ in RSA without treatment (9-13).

In China, the positive rates of ACL and LA were 2.2 and $0.07-0.27 \%$, while those with RSA history were 4.08 and $5.71 \%$ (2013), respectively, and in Western countries (USA, 1995; UK, 1996; Switzerland, 1987; Italy, 1997; Spain, 1994; The Netherlands, 1996), RSA pregnant women have ACL positive rates of 5-51\% (14,15). For the past 30 years, several treatment options have been available, such as aspirin, heparin, plasma exchange, glucocorticoid, immunoglobulin and other combined or single applications for ACL $(16,17)$, but the research conclusions about optimal treatment still remains controversial. At present, heparin combined with aspirin is a widely used treatment $(18,19)$. The aim of the present study was to perform a meta-analysis to elucidate the effects of aspirin and heparin in the treatment of RSA in women with APS and to provide a basis for informed clinical treatment.

\section{Materials and methods}

Search strategy. Previous relevant studies published before May 2019 on the use of aspirin and heparin in the treatment of RSA in women with APS were obtained from the Cochrane (www.cochranelibrary.com), Pubmed (pubmed.ncbi.nlm.nih. gov), Embase (www.embase.com), CNKI (www.cnki.net), VANFUN (www.wanfangdata.com.cn/index.html) and VIP 
(www.cqvip.com) databases. The references of all identified articles were also reviewed to obtain additional studies. Search terms were as follows: 'Antiplatelet', 'anticoagulants', 'lupus', 'antiphospholipid syndrome', 'antiphospholipid antibody', 'recurrent miscarriage', 'recurrent abortion', 'spontaneous fetal loss', 'abortion', 'habitual', 'habitual abortion', 'treatment', 'heparin', 'aspirin', 'randomized', 'randomized controlled trial' and 'RCT'. These terms were used in combination with 'AND' or 'OR'. The present meta-analysis was performed independently by two investigators and disagreements were resolved by a third investigator. The main disagreement was whether one of the selected articles should be included or excluded in the meta-analysis. When the disagreement occurred, the three investigators read the article together and determined whether to incorporate the study.

Following the Participants, Interventions, Comparisons, Outcomes and Study design principle (20), the key search terms were: (P) female with RSA associated with APS; (I) patients in treatment group treated by aspirin and/or heparin; (C) placebo, aspirin and prednisone, intravenous immunoglobulin, prednisone or aspirin alone; $(\mathrm{O})$ fertility outcome indexes, including live birth, pre-term delivery, miscarriage, birth weight, vaginal delivery, cesarean delivery, intrauterine death, gestational age at birth, intrauterine growth restriction (IUGR), gestational diabetes, thrombocytopenia and pre-eclampsia; and (S) randomized controlled trial.

Study selection criteria. All included studies met the following criteria: i) The study was a randomized controlled trial; ii) the research subjects were women with RSA associated with APS; iii) the treatment in the experimental group included aspirin and/or heparin, while the treatment in the control group was not limited; and iv) the study was written in English or Chinese. The exclusion criteria were as follows: i) Repeated articles or results; ii) clear data errors (including wrong index units, inconsistent data, results exceeding maximum values); iii) case reports, case-control studies, theoretical research, conference reports, systematic reviews, meta-analyses, or other forms of research or comments, which were not designed in a randomized controlled manner; and iv) irrelevant outcomes.

Data extraction. For each included study, two categories of information were extracted: Basic information and primary study outcomes. Basic information relevant to the current meta-analysis included authors' names, year of publication, sample size, patients' age and sex, results of the treatment of experimental and control groups, and Jadad score (21). Primary clinical outcomes relevant to the analysis included: Live birth, pre-term delivery, miscarriage, birth weight, vaginal delivery, cesarean delivery, intrauterine death, gestational age at birth, IUGR, gestational diabetes, thrombocytopenia and pre-eclampsia.

Quality assessment. Study quality was determined on the basis of Jadad scores, which were assigned according to the following criteria: i) Whether studies included a specific statement regarding randomization; ii) whether the method used to randomize patients was appropriate; iii) whether the study was conducted in a double-blinded manner; iv) whether the approach to double-blinding was described appropriately; and v) whether patient information was complete. A Jadad score $<3$ was indicative of low quality and therefore associated with a substantial risk of bias. Data extraction was performed independently by two investigators and disagreements were resolved by a third investigator. The main disagreements included: data extraction of primary clinical outcomes and the Jadad score of the included studies. When the data or scores were not consistent, the three investigators read the article together, analyzed and discussed data, and then extracted the data again until a consensus was reached.

Statistical analysis. STATA software (version 10.0; StataCorp LP) was used for all analyses. Heterogeneity in the study results was assessed using $\chi^{2}$ and $\mathrm{I}^{2}$ tests and appropriate analytical models (fixed effect or random effect) were determined accordingly. $\mathrm{P} \leq 0.05$ and an $\mathrm{I}^{2}>50 \%$ indicated high heterogeneity and a random effect model was used in this case. $\mathrm{P}>0.05$ and an $\mathrm{I}^{2} \leq 50 \%$ indicated acceptable heterogeneity and a fixed effect model was used instead. Results for continuous variables are presented as the mean \pm standard deviation and were compared on the basis of weighted mean difference (WMD), while categorical data are presented as percentages and compared based on relative risk (RR) and odds ratio. WMD and 95\% CI were used to analyze the birth weight and gestational age at birth, while RR and 95\% CI were used to analyze live birth, pre-term delivery, miscarriage, vaginal delivery, cesarean delivery, intrauterine death, IUGR, gestational diabetes, thrombocytopenia and pre-eclampsia. Pooled data (WMD or RR with $95 \% \mathrm{CI}$ ) were analyzed to determine the effects of aspirin and heparin treatment on RSA in women with APS. Publication bias was evaluated using the funnel plot method. A symmetrical funnel plot that was narrow at the top and wide at the bottom indicated no publication bias in relation to the analyzed index. Begg's and Mazumdar's rank test was to analyze the direct correlation between standardized effect size and its variance. A value of 0 indicates that there is no direct correlation between effect size and accuracy; while a value higher than 0 indicates that there is a correlation, with an increasing value signifying a stronger correlation. Begg's rank correlation test and Egger's linear regression method were used to determine the possible publication bias through visually inspecting funnel plots. $\mathrm{P}<0.05$ was considered statistically significant.

\section{Results}

Overview of included studies. A total of 1,218 articles were identified, of which 1,147 were excluded by primary title and abstract review. The remaining 71 articles were subject to a complete full-text assessment, which further excluded 49 articles for failing to meet study inclusion criteria and the exclusion reasons were as follows: i) 5 articles with theoretical research; ii) 11 articles with no clinical outcomes, iii) 4 repeated articles; and iv) 29 non-randomized trials. Thus, a total of 22 studies (22-43) were ultimately identified that met the inclusion criteria for the present meta-analysis. These 22 studies involved 1,515 patients in the treatment group and 1,531 in the control group. The study selection process is outlined in Fig. 1.

Table I summarizes basic information for each study, including authors' names, year of publication, sample, age, 
Table I. Basic characteristics of the included studies.

\begin{tabular}{|c|c|c|c|c|c|c|}
\hline \multirow[b]{2}{*}{ Author, year } & \multicolumn{2}{|c|}{ No. of patients } & \multicolumn{2}{|c|}{ Interventions } & \multirow[b]{2}{*}{ Jadad score } & \multirow[b]{2}{*}{ Refs } \\
\hline & $\mathrm{T}$ & $\mathrm{C}$ & $\mathrm{T}$ & $\mathrm{C}$ & & \\
\hline Rai et al, 1997 & 45 & 45 & Heparin and aspirin & Aspirin & 3 & $(25)$ \\
\hline Farquharson et al, 2002 & 51 & 47 & Heparin and aspirin & Aspirin & 5 & $(26)$ \\
\hline Triolo et al, 2003 & 19 & 21 & Heparin and aspirin & Intravenous immunoglobulin & 3 & $(23)$ \\
\hline Goel et al, 2006 & 33 & 39 & Heparin and aspirin & Aspirin & 4 & $(27)$ \\
\hline Dendrinos et al, 2009 & 40 & 38 & Heparin and aspirin & Intravenous immunoglobulin & 3 & $(28)$ \\
\hline Ismail et al, 2016 & 90 & 90 & Heparin and aspirin & Placebo & 3 & $(30)$ \\
\hline Tulppala et al, 1997 & 33 & 33 & Aspirin & Placebo & 5 & $(32)$ \\
\hline Pattison et al, 2000 & 20 & 20 & Aspirin & Placebo & 3 & $(22)$ \\
\hline Cowchock et al, 1992 & 12 & 8 & Heparin & Prednisone & 3 & $(34)$ \\
\hline Laskin et al, 2009 & 45 & 43 & Heparin and aspirin & Aspirin & 5 & $(29)$ \\
\hline Zhou et al, 2012 a & 30 & 31 & Heparin and aspirin & Aspirin & 3 & $(31)$ \\
\hline Zhou et al, 2012 b & 30 & 30 & Heparin and aspirin & Placebo & 3 & $(31)$ \\
\hline Zhang et al, 2015 & 27 & 27 & Heparin & Aspirin and prednisone & 2 & $(33)$ \\
\hline Bu Mingxiu et al, 2009 & 20 & 20 & Heparin & Prednisone & 2 & $(36)$ \\
\hline Jinhua et al, 2003 & 24 & 24 & Heparin & Aspirin and prednisone & 2 & $(35)$ \\
\hline Madani et al, 2019 & 30 & 30 & Aspirin & Placebo & 4 & $(37)$ \\
\hline Blomqvist et al, 2018 & 200 & 200 & Aspirin & Placebo & 4 & $(38)$ \\
\hline Bao et al, 2017 & 497 & 518 & Heparin and aspirin & Aspirin & 4 & $(39)$ \\
\hline Maged et al, 2016 & 90 & 90 & Heparin and aspirin & Placebo & 5 & $(40)$ \\
\hline Zhang et al, 2018 & 44 & 44 & Heparin and aspirin & Aspirin & 3 & $(41)$ \\
\hline Zhaojuan et al, 2018 & 28 & 28 & Heparin and aspirin & Aspirin & 3 & $(42)$ \\
\hline Tang et al, 2012 & 44 & 42 & Heparin and aspirin & Aspirin and prednisone & 3 & $(24)$ \\
\hline Liang et al, 2015 & 63 & 63 & Heparin & Aspirin and prednisone & 3 & $(43)$ \\
\hline
\end{tabular}

$\mathrm{T}$, treatment group; C, control group; Refs., reference.

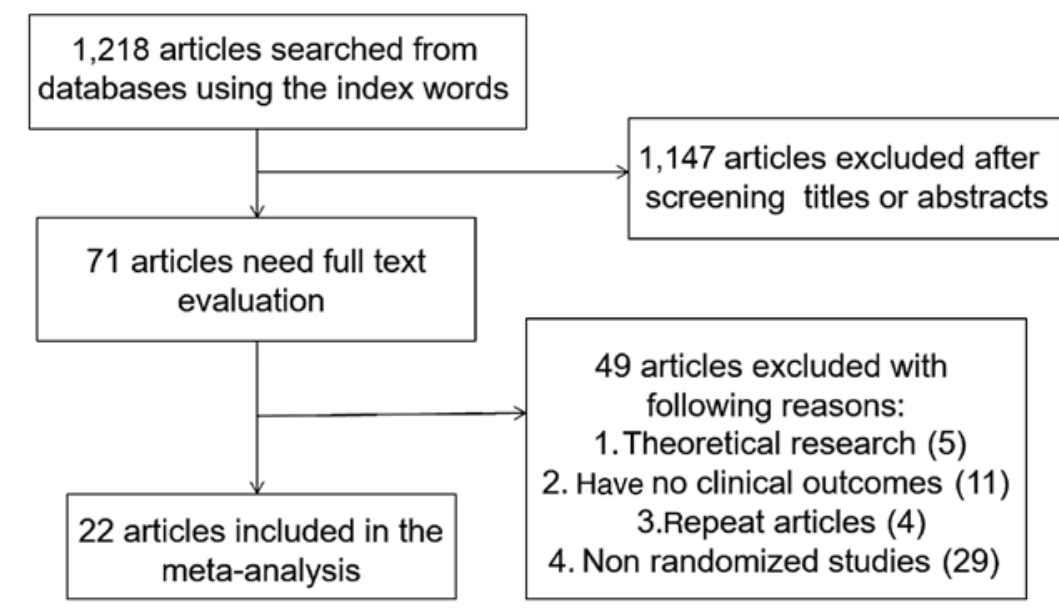

Figure 1. Literature search and selection strategy.

sex, treatment methods and Jadad score. The mean Jadad score for these selected studies was 3.39 (>3), indicating that, on average, these were of high quality.

All the indexes were divided into the following sub-groups for further analysis: Heparin and aspirin vs. aspirin alone, heparin and aspirin vs. intravenous immunoglobulin, heparin and aspirin vs. aspirin and prednisone, heparin and aspirin vs. placebo, aspirin vs. placebo, heparin vs. prednisone and heparin vs. aspirin and prednisone.

Live birth. A total of 20 studies, representing 1,427 patients in the treatment group and 1,442 patients in the control group, reported the live birth rates. Based on a $\chi^{2} \mathrm{P}<0.0001$ and an $\mathrm{I}^{2}=70.9 \%$, a random effect model was used to assess live birth. Overall, the 


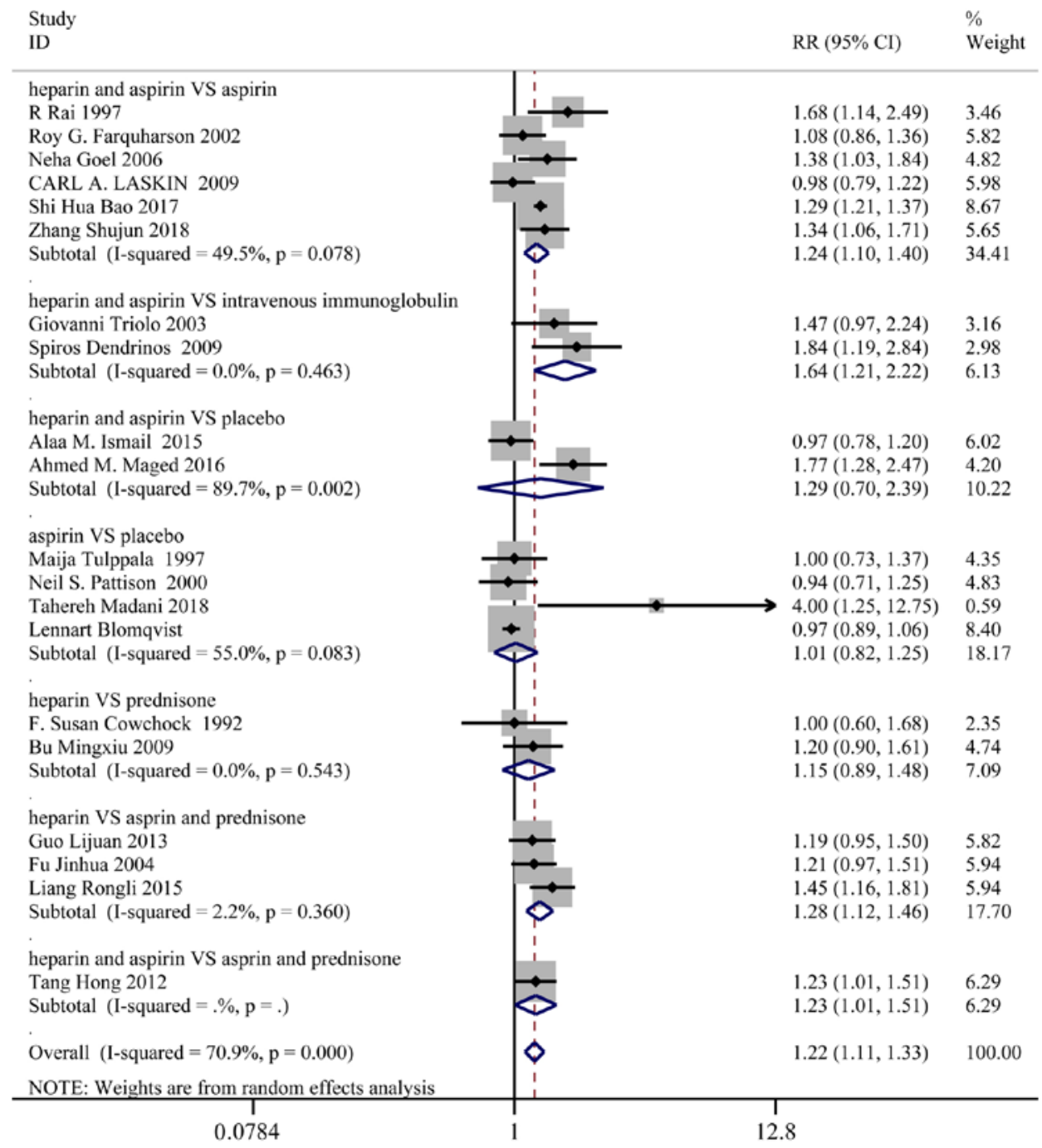

Figure 2. Forest plot for live birth incidence. Data are presented as 'treatment group vs. control group'. Where the RR (95\% CI) of both groups was $>1$, the incidence of live birth was considered significantly higher in the treatment group than the control group. If the RR (95\% CI) of both groups was $<1$, the incidence of live birth was considered significantly lower in the treatment group than the control group. In all other situations, no statistical difference could be inferred from the two groups. RR, relative risk.

live birth rate was significantly higher in the treatment group than the control group (RR: 1.22; 95\% CI: 1.11-1.33). In the sub-group analysis, live birth was markedly improved in the following groups (Fig. 2): Heparin and aspirin vs. aspirin (RR: 1.24; 95\% CI: 1.10-1.40), heparin and aspirin vs. intravenous immunoglobulin (RR: 1.64; 95\% CI: 1.21-2.22), heparin and aspirin vs. aspirin and prednisone (RR: 1.23; 95\% CI: 1.01-1.51), heparin vs. aspirin and prednisone (RR: 1.28; 95\% CI: 1.12-1.46).

Birth weight. A total of 8 studies, with a total of 331 patients in the treatment group and 333 patients in the control group, reported the birth weights. Based on a $\chi^{2} \mathrm{P}<0.0001$ and an $\mathrm{I}^{2}=93.4 \%$, a random effect model was used to assess the birth weight (Fig. 3). No significant difference in birth weight was identified between the two groups [weighted MD (WMD): 154.65 g; 95\% CI: -27.75-337.06]. In the sub-group analysis, birth weight was significantly higher in the heparin and aspirin group than the placebo group (WMD: 708.00; 95\% CI: 531.06-884.94).

Pre-term delivery. A total of 11 studies, with 480 patients in the treatment group and 482 patients in the control group, reported the incidence of pre-term delivery. Based on a $\chi^{2} \mathrm{P}=0.007$ and an $\mathrm{I}^{2}=59.0 \%$, a random effect model was used to assess pre-term delivery (Fig. 4). No significant difference in pre-term delivery was observed between the two groups (RR: $0.72 ; 95 \% \mathrm{CI}$ : $0.40-1.28)$. In the sub-group analysis, the incidence of pre-term delivery was higher in the heparin and aspirin group than the aspirin and prednisone group (RR: 1.39; 95\% CI: 1.03-1.88).

Other results. No significant differences in the rates of cesarean delivery, intrauterine death, gestational diabetes and thrombocytopenia were identified between the two groups.

Vaginal delivery was significantly higher in the heparin and aspirin group than the intravenous immunoglobulin group (RR: 2.07; 95\% CI: 1.19-3.62). The gestational age at birth was markedly higher in the heparin and aspirin group compared with the placebo group (WMD: 4.11 week; 95\% CI: 3.68-4.53).

The incidence of IUGR was lower in the treatment group than the control group (RR: $0.42 ; 95 \% \mathrm{CI}: 0.20-0.88$ ). In the sub-group analysis, the incidence of IUGR was also lower in the heparin and aspirin group compared with the placebo group (RR: 0.33 ; 95\% CI: $0.14-0.80)$. 


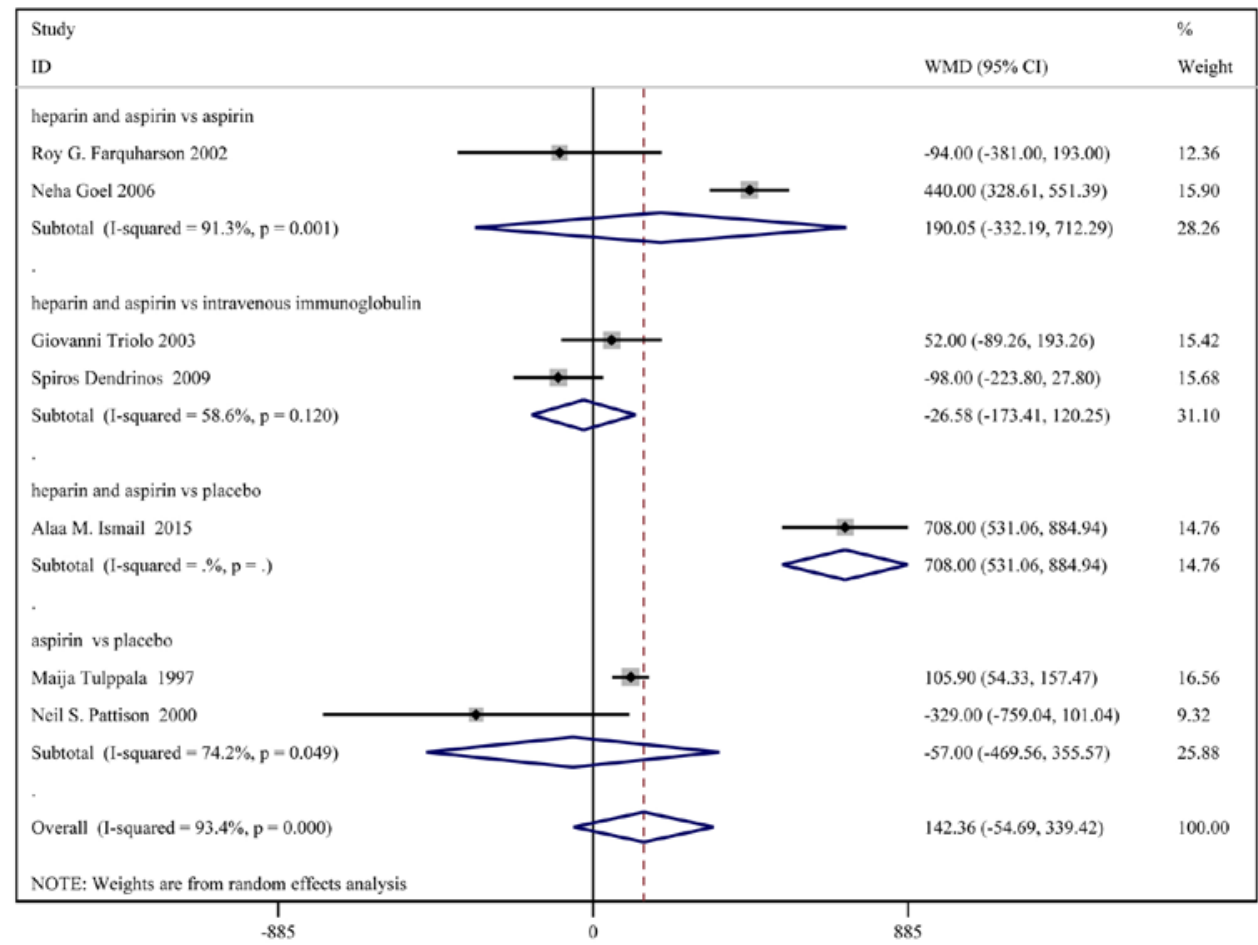

Figure 3. Forest plot for birth weight. Data are presented as 'treatment group vs. control group'. When the WMD (95\% CI) of both groups was $>0$, birth weight was considered significantly higher in the treatment group compared with the control group. If the WMD (95\% CI) of both groups was $<0$, birth weight was considered significantly lower in the treatment group compared with the control group. In all other situations, no statistical difference could be inferred from the 2 groups. WMD, weighted mean difference.

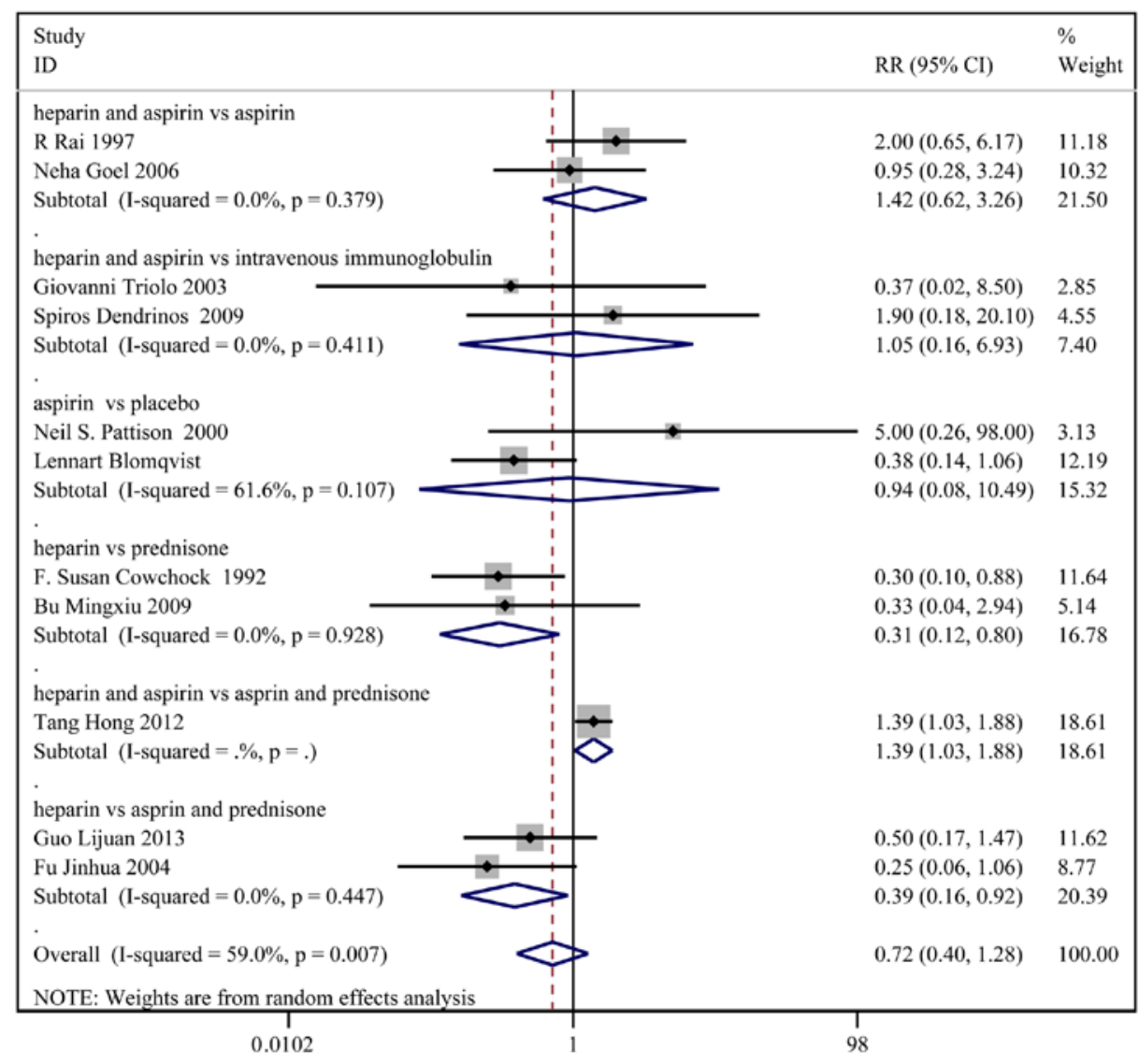

Figure 4. Forest plot for pre-term delivery. Data are presented as 'treatment group vs. control group'. When the RR (95\% CI) of both groups was $>1$, the incidence of pre-term delivery was considered significantly higher in the treatment group compared with the control group. If the RR (95\% CI) of both groups was $<1$, the incidence of pre-term delivery was considered significantly lower in the treatment group compared with the control group. In all other situations, no statistical difference could be inferred from the 2 groups. RR, relative risk. 
Table II. The others results of meta-analysis.

\begin{tabular}{|c|c|c|c|c|c|c|c|}
\hline \multirow[b]{2}{*}{ Index } & \multirow[b]{2}{*}{ Interventions } & \multirow[b]{2}{*}{$\mathrm{RR}(95 \% \mathrm{CI})$} & \multirow[b]{2}{*}{${ }^{\text {b}} \mathrm{P}$-value } & \multirow[b]{2}{*}{$\mathrm{I}^{2}, \%$} & \multirow[b]{2}{*}{${ }^{\mathrm{c} P}$-value } & \multicolumn{2}{|c|}{ P-value } \\
\hline & & & & & & Begg's & Egger's \\
\hline \multirow[t]{3}{*}{ Vaginal delivery } & Overall & $1.37(0.63-3.00)$ & 0.022 & 80.9 & 0.432 & 1.000 & - \\
\hline & HA vs. II & $2.07(1.19-3.62)$ & - & - & 0.011 & - & - \\
\hline & A vs. Pl & $0.95(0.64-1.42)$ & - & - & 0.802 & - & - \\
\hline \multirow[t]{4}{*}{ Cesarean delivery } & Overall & $1.09(0.68-1.75)$ & 0.346 & 10.5 & 0.729 & 0.462 & 0.574 \\
\hline & HA vs. A & $0.75(0.38-1.47)$ & - & - & 0.402 & - & - \\
\hline & HA vs. II & $2.39(0.71-8.05)$ & 0.187 & 42.6 & 0.159 & 0.317 & - \\
\hline & A vs. Pl & $1.13(0.48-2.65)$ & 0.750 & 0.0 & 0.788 & 0.317 & - \\
\hline Intrauterine death & HA vs. II & $0.44(0.07-2.79)$ & 0.387 & 0.0 & 0.382 & 1.000 & - \\
\hline \multirow[t]{5}{*}{ Gestational age at birth } & Overall & $1.24(-0.46-2.93)^{\mathrm{a}}$ & 0.000 & 97.9 & 0.154 & 0.764 & 0.976 \\
\hline & HA vs. A & $0.04(-1.66-1.73)^{\mathrm{a}}$ & 0.000 & 94.7 & 0.967 & 0.602 & 0.936 \\
\hline & HA vs. II & $0.40(-1.00-1.80)^{\mathrm{a}}$ & - & - & 0.577 & - & - \\
\hline & HA vs. Pl & $4.11(3.68-4.53)^{\mathrm{a}}$ & 0.925 & 0.0 & $<0.001$ & 0.317 & - \\
\hline & A vs. Pl & $0.00(-0.49-0.49)^{\mathrm{a}}$ & - & - & 1.000 & - & - \\
\hline \multirow[t]{3}{*}{ IUGR } & Overall & $0.42(0.20-0.88)$ & 0.267 & 18.7 & 0.021 & 1.000 & - \\
\hline & HA vs. Pl & $0.33(0.14-0.80)$ & - & - & 0.014 & - & - \\
\hline & A vs. Pl & $0.89(0.20-3.96)$ & - & - & 0.873 & - & - \\
\hline \multirow[t]{7}{*}{ Miscarriages } & Overall & $0.60(0.49-0.73)$ & 0.033 & 49.2 & 0.000 & 0.436 & 0.437 \\
\hline & HA vs. A & $0.59(0.40-0.87)$ & 0.605 & 0.0 & 0.007 & 0.602 & 0.703 \\
\hline & A vs. Pl & $1.20(0.80-1.81)$ & 0.862 & 0.0 & 0.382 & 0.317 & - \\
\hline & HA vs. Pl & $0.47(0.33-0.67)$ & 0.946 & 0.0 & $<0.001$ & 0.317 & - \\
\hline & HA vs. Apr & $0.35(0.12-1.01)$ & - & - & 0.051 & - & - \\
\hline & H vs. Apr & $0.32(0.17-0.62)$ & 0.962 & 0.0 & 0.001 & 0.317 & - \\
\hline & $\mathrm{H}$ vs. Pl & $0.20(0.03-1.56)$ & - & - & 0.125 & - & - \\
\hline \multicolumn{8}{|l|}{ Gestational diabetes } \\
\hline & Overall & $0.26(0.06-1.14)$ & 0.966 & 0.0 & 0.073 & 1.000 & - \\
\hline & A vs. Pl & $0.25(0.03-2.07)$ & - & - & 0.199 & - & - \\
\hline & H vs. Pl & $0.27(0.03-2.10)$ & - & - & 0.209 & - & \\
\hline \multicolumn{8}{|l|}{ Thrombocytopenia } \\
\hline & Overall & $0.69(0.30-1.57)$ & 0.798 & 0.0 & 0.373 & 0.806 & 0.373 \\
\hline & HA vs. A & $0.51(0.16-1.63)$ & 0.698 & 0.0 & 0.255 & 0.602 & 0.899 \\
\hline & HA vs. Pl & $0.50(0.05-5.22)$ & - & - & 0.563 & - & - \\
\hline & HA vs. Apr & $1.27(0.30-5.35)$ & - & - & 0.742 & - & - \\
\hline \multicolumn{8}{|l|}{ Pre-eclampsia } \\
\hline & Overall & $0.51(0.31-0.87)$ & 0.936 & 0.0 & 0.012 & 0.260 & 0.438 \\
\hline & HA vs. A & $0.52(0.05-5.40)$ & - & - & 0.581 & - & - \\
\hline & HA vs. Pl & $0.48(0.25-0.93)$ & 0.582 & 0.0 & 0.029 & 0.317 & - \\
\hline & A vs. Pl & $0.58(0.24-1.43)$ & 0.666 & 0.0 & 0.240 & 0.602 & 0.643 \\
\hline
\end{tabular}

${ }^{\mathrm{a}}$ Analyzed by WMD $(95 \% \mathrm{CI}) .{ }^{\mathrm{b}} \mathrm{P}$-value of heterogeneity $\chi^{2}$; ${ }^{\mathrm{c}} \mathrm{P}$-value of pooled statistic. H, heparin alone, A, aspirin alone; HA, heparin combined with aspirin; II, intravenous immunoglobulin; Pl, placebo; Pr, prednisone; APr, aspirin and prednisone; WMD, weighted mean difference; RR, relative risk; IUGR, intrauterine growth restriction.

Moreover, the incidence of miscarriages was lower in the treatment group compared with the control group (RR: 0.60 ; 95\% CI: 0.49-0.73), as well as lower in the heparin and aspirin group than the aspirin (RR: $0.59 ; 95 \% \mathrm{CI}: 0.40-0.87$ ) and placebo groups (RR: 0.47; 95\% CI: 0.33-0.67) and lower in the heparin group compared with the aspirin and prednisone groups (RR: 0.32 ; $95 \% \mathrm{CI}$ : 0.17-0.62).
Furthermore, the incidence of pre-eclampsia was lower in the treatment group than the control group (RR: $0.51 ; 95 \% \mathrm{CI}$ : 0.31-0.87) and the placebo group (RR: 0.48; 95\% CI: 0.25-0.93). The aforementioned results are summarized in Table II.

Quality and bias assessment. An assessment of study quality and risk of bias was performed using multiple complementary 


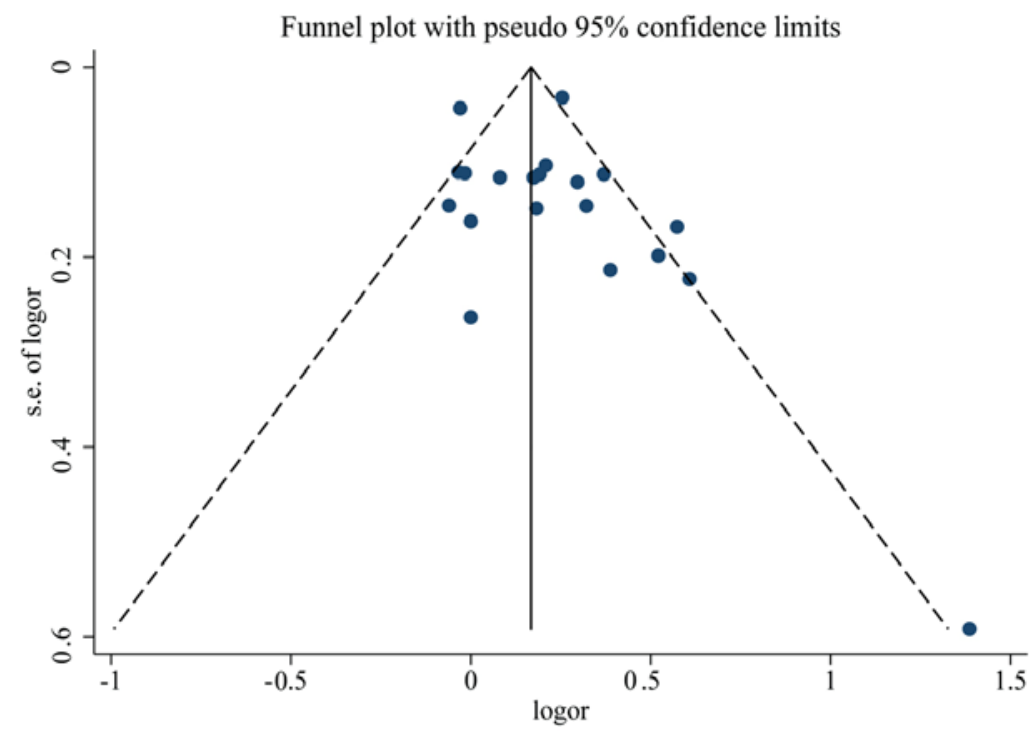

Figure 5. Funnel plot analysis of the included studies. OR, odds ratio.

methods, including funnel plots, Begg's and Mazumdar's rank test, and Egger's test. There was clear symmetry in the log RR funnel plot for included studies of live birth, suggesting a low publication bias risk (Fig. 5). The results of Begg's and Mazumdar's rank test $(\mathrm{Z}=1.52 ; \mathrm{P}=0.127)$ and Egger's test $(\mathrm{P}=0.351)$ demonstrated that there was no significant risk of bias among the studied results.

\section{Discussion}

The mechanisms underlying the effects of heparin on RSA are unclear. However, several possibilities have been suggested in the literature. It has been demonstrated that the combination of heparin and heparin antithrombin III can exert direct anticoagulant effects (44). Alternatively, heparin can combine with antiphospholipid antibody (APA) and reduce its biological activity to protect the phospholipid components of blastocyst trophoblast cells from damage (45). APA can activate the complement system, especially $\mathrm{C} 3$ and $\mathrm{C} 5$, which in turn can promote inflammation and immune responses (46-48). In this respect, heparin can not only reduce inflammation, affect antigen processing, inhibit the formation of antibodies and complement-mediated abnormal immune responses, but also improve the implantation rate of embryos (49). Additionally, heparin can reduce the activity of aspartic acid caspase-3, inhibit the apoptosis of trophoblast cells and participate in the adhesion and invasion of blastocysts to endometrial epithelium, thereby promoting the proliferation of trophoblast cells and the formation of the placenta (50). As heparin has a relatively high molecular weight, it is difficult to pass through the placenta and has no teratogenic effects, thus is safe for the fetus and can be used in early pregnancy (51-53).

Anticoagulant therapy is mainly used for pregnant patients with a history of abortion and positive APA (54). The primary goal of this therapy is to protect the mother from thrombosis and reduce the risk of miscarriage (55). Aspirin can inhibit platelet aggregation, increase prostacyclin levels and has an anticoagulant effect $(56,57)$. Hertz-Picciotto et al (58) observed that only high doses of aspirin (650-2, $600 \mathrm{mg} / \mathrm{day})$ can lead to obvious fetal malformation, while low doses of aspirin ( $<150 \mathrm{mg} /$ day $)$ do not affect the quality of fetal birth or increase perinatal fetal mortality and therefore is safe to use. However, it should be noted that long-term use of aspirin may increase the risk of peptic ulcer disease (59). Therefore, aspirin should be used with caution when coagulation-related indicators are abnormal and APA is positive.

In the present study, it was observed that heparin and aspirin significantly improved live birth, compared with aspirin and prednisone. Compared with the placebo group, the heparin and aspirin group displayed markedly improved gestational age at birth, decreased the incidence of IUGR and miscarriage and a lower incidence of pre-eclampsia. Compared with the aspirin group, the heparin and aspirin group significantly improved live birth and decreased the incidence of miscarriages. Furthermore, heparin and aspirin significantly increased the birth weight relative to placebo. Heparin and aspirin markedly improved live birth and vaginal delivery, compared with intravenous immunoglobulin.

However, there were certain limitations in the present analysis. Indeed, only randomized studies were included and individual studies varied in their exclusion and inclusion criteria, as well as dosage. In addition, only a limited number of studies were included. Lastly, pooled data were analyzed, as individual patient data were not available; therefore, excluding the possibility of a more comprehensive analysis.

Overall, heparin and aspirin may be an optimal combination for treating RSA in women with APS. Nonetheless, the limited number of studies included in the present meta-analysis warrants further validation.

\section{Acknowledgements}

Not applicable.

\section{Funding}

No funding was received. 


\section{Availability of data and materials}

The datasets used and/or analyzed during the current study are available from the corresponding author on reasonable request.

\section{Authors' contributions}

XY made substantial contributions to the conception and design of the current study. XY drafted the article and critically revised the draft for important intellectual content. Data acquisition, analysis and interpretation were performed by LH. All authors read and approved the final manuscript. All authors agreed to be accountable for all aspects of the work and ensuring that questions related to the accuracy or integrity of the work are appropriately investigated and resolved.

\section{Ethics approval and consent to participate}

Not applicable.

\section{Patient consent for publication}

Not applicable.

\section{Competing interests}

The authors declare that they have no competing interests.

\section{References}

1. Linnemann B: Antiphospholipid syndrome - an update. Vasa 47: 451-464, 2018

2. Uthman I, Noureldine MH, Ruiz-Irastorza G and Khamashta M: Management of antiphospholipid syndrome. Ann Rheum Dis 78: 155-161, 2019.

3. Carmi O, Berla M, Shoenfeld Y and Levy Y: Diagnosis and management of catastrophic antiphospholipid syndrome. Expert Rev Hematol 10: 365-374, 2017.

4. Garcia D and Erkan D: Diagnosis and management of the antiphospholipid syndrome. N Engl J Med 379: 1290, 2018.

5. Fujieda Y, Amengual O and Atsumi T: Pathogenic role of antiphospholipid antibodies: An update. Lupus 27: 2012-2013, 2018.

6. McIntyre JA, Coulam CB and Faulk WP: Recurrent spontaneous abortion. Am J Reprod Immunol 21: 100-104, 1989.

7. Subgroup O: Chinese society of obstetrics and gynecology, Chinese medical association. Zhonghua Fu Chan Ke Za Zhi 51: 3-9, 2016.

8. Lin QD and Qiu LH: Pathogenesis, diagnosis, and treatment of recurrent spontaneous abortion with immune type. Front Med China 4: 275-279, 2010

9. Miyakis S, Lockshin MD, Atsumi T, Branch DW, Brey RL, Cervera R, Derksen RH, de Groot PG, Koike T, Meroni PL, et al: International consensus statement on an update of the classification criteria for definite antiphospholipid syndrome (APS). J Thromb Haemost 4: 295-306, 2006.

10. Carrington B, Sacks G and Regan L: Recurrent miscarriage: Pathophysiology and outcome. Curr Opin Obstet Gynecol 17: 591-597, 2005.

11. Kuon RJ, Wallwiener LM, Germeyer A, Strowitzki T, Daniel V and Toth B: Establishment of a standardized immunological diagnostic procedure in RM patients. J Reprod Immunol 1: 55, 2012.

12. Christiansen OB: Evidence-based investigations and treatments of recurrent pregnancy loss. Curr Opin Obstet Gynecol 18: 304-312, 2006

13. Regan L and Rai R: Epidemiology and the medical causes of miscarriage. Best Pract Res Clin Obstet Gynaecol 14: 839-854, 2000.
14. VinatierD, Dufour P, Cosson Mand Houpeau JL: Antiphospholipid syndrome and recurrent miscarriages. Eur J Obstet Gynecol Reprod Biol 96: 37-50, 2001.

15. Qiujuan Wang and Wenhua Xu: Pregnancy complicated with antiphospholipid syndrome. Zhong Hua Fu Chan Ke Za Zhi Bian Ji Bu 30: 117-119, 1995 (In Chinese).

16. Cervera R: Antiphospholipid syndrome. Thromb Res 151 (Suppl 1): S43-S47, 2017.

17. Schreiber K and Hunt BJ: Managing antiphospholipid syndrome in pregnancy. Thromb Res 181 (Suppl 1): S41-S46, 2019.

18. Del Ross T, Ruffatti A, Visentin MS, Tonello M, Calligaro A, Favaro M, Hoxha A and Punzi L: Treatment of 139 pregnancies in antiphospholipid-positive women not fulfilling criteria for antiphospholipid syndrome: A retrospective study. J Rheumatol 40: 425-429, 2013.

19. Rai RS, Clifford K, Cohen H and Regan L: High prospective fetal loss rate in untreated pregnancies of women with recurrent miscarriage and antiphospholipid antibodies. Hum Reprod 10: 3301-3304, 1995.

20. Zeng X, Sun Z and Tang H: Series ten of meta-analysis: Formulation of eligibility criteria. Chin J Evid Based Cardiovasc Med 5: 6-9, 2013.

21. McCormick F, Cvetanovich GL, Kim JM, Harris JD, Gupta AK, Abrams GD, Romeo AA and Provencher MT: An assessment of the quality of rotator cuff randomized controlled trials: Utilizing the Jadad score and CONSORT criteria. J Shoulder Elbow Surg 22: 1180-1185, 2013.

22. Pattison NS, Chamley LW, Birdsall M, Zanderigo AM, Liddell HS and McDougall J: Does aspirin have a role in improving pregnancy outcome for women with the antiphospholipid syndrome? A randomized controlled trial. Am J Obstet Gynecol 183: 1008-1012, 2000.

23. Triolo G, Ferrante A, Ciccia F, Accardo-Palumbo A, Perino A, Castelli A, Giarratano A and Licata G: Randomized study of subcutaneous low molecular weight heparin plus aspirin versus intravenous immunoglobulin in the treatment of recurrent fetal loss associated with antiphospholipid antibodies. Arthritis Rheum 48: 728-731, 2003.

24. Tang H, Huang MY and Yi T: Effect of low molecular weight heparin combined with low-dose aspirin for ACA positive recurrent spontaneous abortion. J Hainan Med 19-20, 2012.

25. Rai R, Cohen H, Dave M and Regan L: Randomised controlled trial of aspirin and aspirin plus heparin in pregnant women with recurrent miscarriage associated with phospholipid antibodies (or antiphospholipid antibodies). BMJ 314: 253-257, 1997.

26. Farquharson RG, Quenby S and Greaves M: Antiphospholipid syndrome in pregnancy: A randomized, controlled trial of treatment. Obstet Gynecol 100: 408-413, 2002.

27. Goel N, Tuli A and Choudhry R: The role of aspirin versus aspirin and heparin in cases of recurrent abortions with raised anticardiolipin antibodies. Med Sci Monit 12: CR132-CR136, 2006.

28. Dendrinos S, Sakkas E and Makrakis E: Low-molecular-weight heparin versus intravenous immunoglobulin for recurrent abortion associated with antiphospholipid antibody syndrome. Int J Gynaecol Obstet 104: 223-225, 2009.

29. Laskin CA, Spitzer KA, Clark CA, Crowther MR, Ginsberg JS, Hawker GA, Kingdom JC, Barrett J and Gent M: Low molecular weight heparin and aspirin for recurrent pregnancy loss: Results from the randomized, controlled HepASA Trial. J Rheumatol 36: 279-287, 2009.

30. Ismail AM, Hamed AH, Saso S, Abu-Elhasan AM, Abu-Elghar MM and Abdelmeged AN: Randomized controlled study of pre-conception thromboprophylaxis among patients with recurrent spontaneous abortion related to antiphospholipid syndrome. Int J Gynaecol Obstet 132: 219-223, 2016.

31. Zhou X: The effect of aspirin combined with low molecular weight heparin in treatment of 30 patients with habitual abortion and antiphospholipid syndrome. Cn Mod Doct 50: 60-62, 2012 .

32. Tulppala M, Marttunen M, Söderstrom-Anttila V, Foudila T, Ailus K, Palosuo T and Ylikorkala O: Low-dose aspirin in prevention of miscarriage in women with unexplained or autoimmune related recurrent miscarriage: Effect on prostacyclin and thromboxane A2 production. Hum Reprod 12: 1567-1572, 1997.

33. Zhang $\mathrm{G}$ and Cheng L: Effect of low molecular weight heparin combined with low dose aspirin on hormone level and immune function in patients with recurrent spontaneous abortion. Zhongguo Sheng Hua Yao Wu Za Zhi 67-69, 2015 (In Chinese). 
34. Cowchock FS, Reece EA, Balaban D, Branch DW and Plouffe L: Repeated fetal losses associated with antiphospholipid antibodies: A collaborative randomized trial comparing prednisone with low-dose heparin treatment. Am J Obstet Gynecol 166: 1318-1323, 1992.

35. Jinhua F, Wang Z and Lang F: Study of heparin on pregnant woman with recurrent pregnant loss and positive anti-phospholipid antibody. Zhonghua Wei Chan Yi Xue Za Zhi 2003.

36. Clinical observation on 40 autoimmune habitual abortion patients with low molecular weight heparin calcium. China Practical Medicine 2009.

37. Madani T, Ahmadi F, Jahangiri N, Bahmanabadi A and Bagheri Lankarani N: Does low-dose aspirin improve pregnancy rate in women undergoing frozen-thawed embryo transfer cycle? A pilot double-blind, randomized placebo-controlled trial. J Obstet Gynaecol Res 45: 156-163, 2019.

38. Blomqvist L, Hellgren M and Strandell A: Acetylsalicylic acid does not prevent first-trimester unexplained recurrent pregnancy loss: A randomized controlled trial. Acta Obstet Gynecol Scand 97: 1365-1372, 2018

39. Bao SH, Sheng SL, Liao H, Zhou Q, Frempong ST and Tu WY: Use of D-dimer measurement to guide anticoagulant treatment in recurrent pregnancy loss associated with antiphospholipid syndrome. Am J Reprod Immunol 78: 78, 2017.

40. Maged AM, Abdelhafiz A, Mostafa WA, El-Nassery N, Fouad M, Salah E and Kotb A: The role of prophylactic use of low dose aspirin and calheparin in patients with unexplained recurrent abortion. Gynecol Endocrinol 32: 970-972, 2016.

41. Zhang Shuyun ZH: Clinical analysis of low molecular weight heparin and aspirin in treating recurrent pregnancy loss caused by antiphospholipid antibodies. Hebei Med 24: 422-426, 2018.

42. Zhaojuan S: Evaluation of the efficacy of low molecular weight heparin combined with aspirin in the treatment of recurrent abortion caused by anti-phospholipid antibody. J Pract Gynecol Endocrinol 5: 53-54, 2018.

43. Liang RH: Effect of low molecular weight heparin combined with Zishen Yutai pill for RSA caused by positive ACA. Chin Youjiang Med J 43: 471-473, 2015.

44. Schreiber K, Sciascia S, de Groot PG, Devreese K, Jacobsen S, Ruiz-Irastorza G, Salmon JE, Shoenfeld Y, Shovman O and Hunt BJ: Antiphospholipid syndrome. Nat Rev Dis Primers 4: $17103,2018$.

45. Galli $M$ and Barbui T: Antiphospholipid antibodies and pregnancy. Best Pract Res Clin Haematol 16: 211-225, 2003.

46. D'Ippolito S, Di Simone N, Di Nicuolo F, Castellani R and Caruso A: Antiphospholipid antibodies: Effects on trophoblast and endothelial cells. Am J Reprod Immunol 58: 150-158, 2007.

47. Katsuragawa $H$, Kanzaki $H$, Inoue $T$, Hirano $T$, Mori $T$ and Rote NS: Monoclonal antibody against phosphatidylserine inhibits in vitro human trophoblastic hormone production and invasion. Biol Reprod 56: 50-58, 1997.
48. Di Simone N, Raschi E, Testoni C, Castellani R, D'Asta M, Shi T, Krilis SA, Caruso A and Meroni PL: Pathogenic role of anti-beta 2-glycoprotein I antibodies in antiphospholipid associated fetal loss: Characterisation of beta 2-glycoprotein I binding to trophoblast cells and functional effects of anti-beta 2-glycoprotein I antibodies in vitro. Ann Rheum Dis 64: 462-467, 2005

49. Chamley LW: Antiphospholipid antibodies: Biological basis and prospects for treatment. J Reprod Immunol 57: 185-202, 2002.

50. Lopez-Pedrera CH, Aguirre MA, Ruiz-Limon P, Pérez-Sánchez C, Jimenez-Gomez Y,Barbarroja NandCuadradoMJ:Immunotherapy in antiphospholipid syndrome. Int Immunopharmacol 27: 200-208, 2015.

51. Sebire NJ, Fox H, Backos M, Rai R, Paterson C and Regan L: Defective endovascular trophoblast invasion in primary antiphospholipid antibody syndrome-associated early pregnancy failure. Hum Reprod 17: 1067-1071, 2002.

52. Franklin RD and Kutteh WH: Effects of unfractionated and low molecular weight heparin on antiphospholipid antibody binding in vitro. Obstet Gynecol 101: 455-462, 2003.

53. Girardi G: Heparin treatment in pregnancy loss: Potential therapeutic benefits beyond anticogulation. J Reprod Lmmunol 66 45-51, 2005.

54. Bates SM, Greer IA, Middeldorp S, Veenstra DL, Prabulos AM and Vandvik PO: VTE, thrombophilia, antithrombotic therapy, and pregnancy: Antithrombotic therapy and prevention of thrombosis, 9th Ed: American College of Chest Physicians Evidence-Based Clinical Practice Guidelines. Chest 141 (Suppl 2): e691S-e736S, 2012.

55. Makino A and Sugiura-Ogasawara M: Anticoagulant therapy and pregnancy. Reprod Med Biol 7: 1-10, 2008.

56. Girardi G, Redecha P and Salmon JE: Hepafin prevents antiphospholipid antibody-induced fetal loss by inhibiting complement activation. Nat Med 10: 1222-1226, 2004.

57. Hills FA, Abrahams VM, González-Timón B, Francis J, Cloke B, Hinkson L, Rai R, Mor G, Regan L, Sullivan M, et al: Heparin prevents programmed cell death in human trophoblast. Mol Hum Reprod 12: 237-243, 2006.

58. Hertz-Picciotto I, Hopenhayn-Rich C, Golub M and Hooper K: The risks and benefits of taking aspirin during pregnancy. Epidemiol Rev 12: 108-148, 1990.

59. Shim YK and Kim N: Nonsteroidal anti-inflammatory drug and aspirin-induced peptic ulcer disease. Korean J Gastroenterol 67: 300-312, 2016.

This work is licensed under a Creative Commons Attribution-NonCommercial-NoDerivatives 4.0 International (CC BY-NC-ND 4.0) License. 